A N N A L E S Annales de Bretagne et des Pays de l'Ouest

\title{
Landévennec 818-2018. Une abbaye bénédictine en
}

Bretagne

\section{André-Yves Bourgès}

\section{(2) OpenEdition}

1 Journals

Édition électronique

URL : https://journals.openedition.org/abpo/7119

DOI : 10.4000/abpo.7119

ISSN : 2108-6443

Éditeur

Presses universitaires de Rennes

\section{Édition imprimée}

Date de publication : 29 septembre 2021

Pagination : 219-222

ISSN : 0399-0826

\section{Référence électronique}

André-Yves Bourgès, «Landévennec 818-2018. Une abbaye bénédictine en Bretagne », Annales de

Bretagne et des Pays de l'Ouest [En ligne], 128-3 | 2021, mis en ligne le 29 septembre 2021, consulté le 11 octobre 2022. URL : http://journals.openedition.org/abpo/7119; DOI : https://doi.org/10.4000/ abpo.7119 
plus peuplée des pays poitevins et charentais. Enfin, les autorités royales auraient longtemps accordé une importance particulière à une ville dont elles eurent plusieurs fois l'occasion d'apprécier la position géostratégique : pendant le conflit qui opposa les Capétiens aux Plantagenêts, pendant la guerre de Cent Ans où elle resta fidèle aux Valois, et au moment des guerres de Religion où cette cité catholique servit de base pour lutter contre les protestants du Poitou et des pays charentais. Toutefois, Poitiers ne se contenta pas de tirer profit de ses atouts pour défendre ses intérêts. Elle s'opposa résolument, et avec constance, à la promotion d'autres villes du Centre Ouest en recourant à ses relations en haut-lieu et en n'hésitant pas à verser des sommes élevées à la monarchie pour acheter sa complicité. Ainsi, à l'époque moderne, elle empêcha la fondation d'une université à Angoulême et l'instauration d'un présidial à Niort et à Fontenay-le-Comte. Elle ne put cependant pas dissuader Louis XIV de créer en 1694 la généralité de La Rochelle, bien que l'élection de La Rochelle fît partie de la généralité de Poitiers.

Poitiers connut un déclassement à partir du XVII ${ }^{\mathrm{e}}$ siècle. Après le plein rétablissement des études juridiques à Paris en 1679, de nombreux étudiants parisiens cessèrent de fréquenter la faculté de droit de Poitiers et l'université perdit de son lustre. La Révocation de l'édit de Nantes priva la ville du rôle central qu'elle avait joué dans le Centre Ouest dans la lutte contre les protestants, et la monarchie lui accorda désormais moins d'attention. Enfin, elle ne fut jamais un centre économique de dimension nationale à cause, notamment, de l'absence d'une voie d'eau lui permettant d'être reliée aux grands courants d'échanges du pays. La torpeur de l'économie ne cessa que dans les années 1960-1980. Le chapitre consacré au début du XXl ${ }^{\mathrm{e}}$ siècle montre qu'en dépit de cette évolution, Poitiers conserve des atouts. Le secteur tertiaire public (enseignement, administration publique, santé, hébergement médicosocial et social) est, certes, surreprésenté, car il représente $40 \%$ de l'emploi local, mais depuis 1975, à quelques exceptions près, les taux de croissance de l'emploi et de la population de Poitiers sont supérieurs à la moyenne nationale, et la ville et sa périphérie accueillent aujourd'hui de gros employeurs privés (activités commerciales, centres d'appel, production d'électricité, fabrication de piles et d'accumulateurs électriques, le Futuroscope).

Les dernières pages de l'étude envisagent le futur de la ville. Elles rappellent que si Poitiers n'est plus une capitale régionale et n'a pas de vocation industrielle, elle peut faire valoir des compétences dans les domaines de la santé, de l'enseignement et de l'action sociale qui sont reconnues au-delà des limites du département de la Vienne. L'avenir dira si le départ de nombreuses directions régionales à partir de 2016 fut une rupture durable ou éphémère dans la longue histoire qu'elle a entretenue avec les pays poitevins et charentais.

François Brizay

CoAtivy, Yves (dir.), Landévennec 818-2018. Une abbaye bénédictine en Bretagne. Actes du colloque de Landévennec des 6, 7 et 8 juin 2018, Brest, CRBC, 2020, 344 p.

Cet ouvrage s'inscrit dans une longue lignée de publications et de travaux sur Landévennec qui, compte tenu de la nature de la documentation conservée, se rapportent souvent aux temps les plus anciens de l'abbaye : il ambitionne pour sa part de couvrir, comme l'indiquent les dates qui figurent dans son titre, la plus grande partie de l'histoire de ce lieu, du IX ${ }^{\mathrm{e}}$ siècle à nos jours; mais en réalité, le Moyen Âge, et plus particulièrement le haut Moyen Âge, se taille une nouvelle fois la part du lion, car l'on voit que plus de la moitié du volume lui est consacrée, contre moins du tiers pour l'époque moderne et un sixième environ pour la période contemporaine. 
L'ouvrage s'ouvre sur la riche contribution, de nature essentiellement archéologique, d'Annie Bardel, Ronan Pérennec et Véronique Bardel, qui plonge le lecteur dans le passé le plus lointain du site monastique, vers 500 environ, et en suit les destinées jusqu'au XIII ${ }^{\mathrm{e}}$ siècle (p. 11-46) : le chantier des fouilles conduites sur place pendant près de quarante années permet notamment de constater que les contraintes naturelles du terrain ont laissé place à partir du $\mathrm{IX}^{\mathrm{e}}$ siècle à des principes de construction influencés par la règle de saint Benoît. Sans atteindre l'équilibre du plan de Saint-Gall, dont Ernst Tremp souligne au demeurant que, loin de représenter l'idéal réformateur de Benoît d'Aniane, " il est bien plutôt une réponse à cette rigueur réformatrice " (p. 125-139), l'évolution observée à Landévennec témoigne donc de la normalisation carolingienne intervenue sur place à cette époque; normalisation sans doute renforcée par "l'intervention d'“envoyés monastiques et canoniaux" ", dont Guy Jarousseau met en lumière le rôle essentiel " au sein des monastères au temps de Louis le Pieux" (p. 141-163). L'impeccable synthèse de Michel Sot sur " l'année 818 dans l'empire carolingien " (p. 47-54) permet de contextualiser l'expédition menée en Bretagne contre le roi Murman - campagne tout autant militaire que religieuse, dont Jehanne Roul (r)établit les circonstances à travers une lecture renouvelée du poème d'Ermold le Noir (p. 67-77) - ainsi que la rencontre de l'empereur avec l'abbé de Landévennec, Matmonoc, rapportée dans un acte donné à cette occasion. Cet acte, connu par la transcription de Wrdisten vers 870 dans sa vita de Guénolé, fait l'objet d'un examen minutieux par Michèle Gaillard, qui en relève les irrégularités (p. 55-66). Si cette chercheuse ne met pas en doute la rencontre de 818 , elle suggère cependant, entre autres hypothèses, que l'acte a pu être "forgé un peu plus tard à partir d'un acte authentique, qui pouvait porter vraisemblablement sur l'octroi de la libre élection de l'abbé, comme c'est le cas dans la grande majorité des cas où un acte de Louis le Pieux mentionne la règle de saint Benoît ": se pose en conséquence la question de la sincérité de certains passages, notamment celui qui concerne les fameux usages scotiques à Landévennec et leur prohibition par l'empereur. Ces interrogations font écho à la stimulante " mise au point " de Jean-Michel Picard " sur les coutumes monastiques irlandaises du haut Moyen Âge " (p. 113-124), qui montre " que la tonsure et les usages de Landévennec ont plus probablement une origine bretonne qu'une origine irlandaise " : l'attribution aux Irlandais serait ainsi " un trait de génie de la part du, ou des rédacteurs de la lettre recopiée ou adaptée par Gurdisten ", car cela évitait d'imputer aux moines du lieu la responsabilité de leurs errements passés. Le corollaire de cette démonstration, nous semble-t-il, c'est que l'influence de l'Irlande en Bretagne continentale, singulièrement à Landévennec, n'a peut-être pas revêtu l'importance que certains lui prêtent. Caroline Brett s'intéresse à la dimension hagiographique qui, au-delà du seul dossier littéraire de Guénolé et Ethbin, lui paraît caractériser l'ensemble du cartulaire de l'abbaye, " dont le but est plutôt commémoratif " (p. 79-86) : dans le cas des supposées donations faites à Guénolé, elle suggère même un rapprochement avec certaines compositions insulaires, en particulier le Livre d'Armagh; mais, au-delà de réserves comme celles que nous venons d'exprimer à propos d'une possible influence irlandaise, cette chercheuse rappelle loyalement qu'Hubert Guillotel et Joëlle Quaghebeur naguère et Cyprien Henry récemment ont préféré reconnaître derrière les actes les plus sommaires du cartulaire de Landévennec le modèle de la " pancarte ", tel qu'il a été mis en œuvre à Locmaria-Quimper par exemple. Stéphane Lebecq s'interroge sur les rapports entre Landévennec, la mer et l'outre-Manche, depuis la fondation de l'abbaye jusqu'à sa destruction par les Vikings, suivi de l'exil de ses moines à Montreuil-surMer, apparemment en vue d'un passage en Grande-Bretagne qui n'a pas eu lieu (p. 87-95) : si le caractère fluvio-maritime (mare flumineum) de son implantation a 
nécessairement joué un rôle dans les destinées du monastère, force est de constater que la principale source à disposition des historiens, à savoir son cartulaire, se montre plutôt avare d'informations à ce sujet. Joëlle Quaghebeur envisage la possibilité que l'abbaye de Landévennec ait pu jouer le rôle de sanctuaire, voire de nécropole, des rois de Bretagne (p. 97-109) : elle dresse à cette occasion un tableau généalogique renouvelé de la dynastie royale, dans lequel Murman, le monarque tant déprécié dans le poème d'Ermold le Noir, est présenté comme le père de Nominoë. L'hypothèse d'une parenté est assurément plausible; mais elle nous semble concerner moins Landévennec que les confins du Poher et du Vannetais occidental où cette dynastie paraît bien avoir été originellement implantée.

Les lieux et les hommes en rapport avec l'abbaye dans la seconde partie du Moyen Âge ont retenu l'attention de plusieurs contributeurs : Julien Bachelier s'est ainsi employé à établir la nomenclature des prieurés de Landévennec (p. 165-185) et Marjolaine Lemeillat s'est efforcée de dresser la courte liste des " gens de savoir " figurant à cette époque parmi les moines du lieu (p. 187-193). Dans les deux cas, ce travail de récolement s'est trouvé limité par le déficit des sources; mais ses résultats s'avèrent d'autant mieux étayés que la documentation concernée, plus aride, si elle n'exclut pas la part de l'hypothèse, ne la favorise guère et cantonne les conclusions aux seules données factuelles. La contribution de Cédric Jeanneau sur l'abbaye de Talmont, qui jette sur ces questions un éclairage extra-breton, vient conforter ce type d'approche qui, à bien des égards, mérite d'être qualifié de technique (p. 205-221). La transition entre le Moyen Âge et l'époque moderne est assurée par l'étude de Guénolé Ridoux qui confronte sources écrites et données archéologiques relatives à l'intrusion de laïcs dans la clôture monastique, en particulier lors de trois épisodes violents intervenus respectivement au moment des incursions scandinaves, durant la guerre de succession de Bretagne et pendant les troubles de la Ligue; mais il est également question sous la plume de l'auteur de " pieux visiteurs ", plus discrets, que l'approche méthodologique retenue permet, pour la période ancienne, de faire sortir un peu de l'ombre (p. 195-203).

Inaugurant la partie de l'ouvrage consacrée à l'histoire de Landévennec " au temps des Mauristes ", Dom Thierry Barbeau expose la place et le rôle, au sein de la nouvelle province mauriste de Bretagne, fondée en 1628, de la vieille abbaye cornouaillaise, qui se réforme dès le début des années 1630 (p. 225-245) : il faut souligner la richesse et la clarté des informations mises à disposition par l'auteur dans les 3 tableaux annexés à son étude, tel celui qui contient les éléments constitutifs d'une véritable base de données prosopographiques sur les prieurs du lieu. Inversant la focale, Daniel-Odon Hurel s'intéresse à la personnalité et aux méthodes de travail de Dom Noël Mars, premier historien de l'abbaye (p. 255-264), notamment ce qui concerne son recours à " toute une série d'ouvrages et outils de travail, [...] sorte de bibliothèque idéale " qu'il est instructif de comparer à " la bibliothèque mauriste ", dont Louis Chevalier donne une description dynamique et évoque la destinée jusqu'après la Révolution (p. 247-253). La contribution de Pierre-Marie Sallé (p. 265-275) met en évidence que l'église abbatiale a fait l'objet, tout au long de la période mauriste, d'une attention particulière de la part des moines. Tandis que la réforme insistait sur l'importance de la clôture, on note que l'organisation de la circulation dans l'édifice continuait de permettre un accès éventuel des laïcs, non seulement au cénotaphe de Guénolé, mais aussi au tombeau de Gradlon, présenté comme le fondateur de l'abbaye : témoignage tangible que le récit des origines renouvelé au bas Moyen Âge avait été avalisé par la communauté réformée. Enfin, Georges Provost montre quel profit tirer de l'exploitation d'un fonds d'archives domestiques, assez méconnues et dont les considérations peuvent apparaître presque triviales, lorsqu'il est question par exemple d'approvisionnements alimen- 
taires, pour mieux connaître d'une inflexion de l'observance de la règle monastique à Landévennec à la fin de l'Ancien régime, phénomène également perceptible dans de nombreux autres monastères (p. 277-292).

La dernière partie de l'ouvrage est intitulée "Crises et reconstructions " : elle s'ouvre par un beau portrait de moine contemporain, celui du père Maurice Guillerm (1901-1955), dressé par Jean-Pascal Gay (p. 295-310), tentative réussie, dans une perspective revendiquée de " micro-histoire ", de reconstitution d'un itinéraire spirituel, servie par une documentation écrite abondante et qui débouche sur la peinture plus large d'un type de monachisme "topique ", paisible, sans véritable dynamique communautaire, qui, dans son absence d'originalité, s'avère porteur à son insu d'orages à venir. Ceux-ci éclatent dans la contribution d'Yvon Tranvouez (p. 311-320), lequel apporte son éclairage de spécialiste sur la manière dont la nouvelle abbaye de Landévennec, depuis sa restauration in situ, a vécu l'évolution et traversé la crise du catholicisme français des décennies 60 et 70 : les témoignages recueillis auprès de religieux ou d'anciens religieux qui ont connu ou furent acteurs de ces événements donnent au récit de l'historien une intéressante coloration mémorielle. Danièle Hervieu-Léger situe délibérément son propos dans le " contexte post-chrétien " qui caractérise aujourd'hui la France et les pays occidentaux : à partir de l'observation des "styles monastiques contemporains ", elle invite prudemment à une démarche " prospective " sur ce que pourrait être l'avenir de l'institution (p. 321-329). Dans l'ultime contribution de l'ouvrage, Michael Jones rend hommage à un bienfaiteur de Landévennec, le docteur Louis Lebreton (1911-1991), et s'efforce de remonter aux fonds d'origine des très nombreux ouvrages, tant imprimés que manuscrits, que ce généreux bibliophile a donné à la communauté et qui ont formé l'embryon, sinon même la plus grande partie des collections de la bibliothèque bretonne de la nouvelle abbaye (p. 331-342) : sans doute s'agissait-il pour le donateur de contribuer à doter Landévennec des ressources littéraires dont tout monastère doit être pourvu... Monasterium sine armario, quasi castrum sine armamentario. Nul doute que l'ouvrage ici recensé aura toute sa place dans cet " arsenal "!

André-Yves Bourgès

Chauou, Amaury, Les Plantagenêts et leur cour, 1154-1216, Paris, Presses universitaires de France/Humensis, 2019, 405 p.

Le nombre de publications sur la cour Plantagenêt ne cesse de croître chaque année, tout particulièrement celles en langue anglaise, comme l'explique l'auteur dans sa sélection bibliographique. Ce livre est une synthèse particulièrement bienvenue, qui met à la disposition du grand public les développements les plus récents en la matière, dont ceux consacrés par Amaury Chauou lui-même aux "représentations et l'imaginaire du pouvoir" (p. 22).

La cour est ici étudiée dans le but d'analyser le pouvoir des souverains Plantagenêts. Les Plantagenêts et leur cour n'est ni une biographie royale ni un récit de la splendeur puis de la chute des Plantagenêts : cet essai se place plutôt dans le renouveau de l'histoire politique post-Annales, ou d'une " histoire $d u$ politique " (p. 21). Le concept de cour royale a tendance à être perçu comme évident : " la cour " est ainsi un concept utile si l'on ne souhaite pas avoir recours à une personnalisation excessive du pouvoir monarchique. Mais jusqu'où les limites de la cour peuvent-elles s'étendre? Qui peut être autorisé à en faire partie? Qu'attend-on exactement de ses membres, en termes d'offices et de fonctions? De façon très pertinente, A. Chauou choisit de ne pas toucher à ce concept nébuleux, ne tente ni de le préciser ni d'imposer des limitations forcément artificielles aux activités et au 